\title{
Analysis of Effect of Ballistic Coefficient in the Formulations and Performance of EKF with Emphasis on Air Drag
}

\author{
A. Sampath Dakshina Murthy ${ }^{1 *}$, S. Koteswara Rao ${ }^{2}$, A. Naga Jyothi ${ }^{1}$ and Rudra Pratap Das ${ }^{1}$ \\ 'Department of ECE, Vignan's Institute of Information Technology, Visakhapatnam - 531162, Andhra Pradesh, India; \\ 2Department of ECE, K. L. University, Vaddeswaram, Guntur District - 522502, Andhra Pradesh, India; \\ sampathdakshinamurthy@gmail.com,rao.sk9@gmail.com,aggala.jyothi@gmail.com, \\ drrudrapratapdas@yahoo.com
}

\begin{abstract}
In one dimensional vertical motion in the case of tracking a target, many types of filters included Kalman varieties have been used so far. This paper is presenting a new approach of inverse ballistic coefficient included in the target state vector for reducing the convergence time. For analysis, estimation of object's altitude and velocity is done by simulation based on noisy error environment measurements. The important of beta (ballistic coefficient) for non linear studies on extended Kalman filter has been established with proper evidence. This work can be further extended to consider the effect of hang off errors and air drag.
\end{abstract}

Keywords:- Air Drag, Altitude, Beta Coefficient, Extended Kalman Filter, Hang Off Error, Inverse Beta, Velocity

\section{Introduction}

The KF is a best situation estimator on behalf of linear method. The EKF is a suboptimal description of the KF design to deal with nonlinearities. However, for mathematical model of systems in engineering, particle is often nonlinear, such as the guidance and control system of rocket, navigation system of aircraft and attitude determination and positioning system with satellites. To improve performance of $\mathrm{EKF}^{1}$, Taylor series expansion is used, for the falling high velocity body to process design of EKF Gordon et.al. ${ }^{2}$ The object entering into the reentry phase and assumed with the purpose of the ballistic coefficient of the object path is unknown. Here the frictional force acts on the objectas it travels through air resistance.

The choice of states in a EKF can show a major dissimilarity, with the intention of the nonlinear discrepancy equation recitation the acceleration on the particular object. The ballistic coefficient, it seems to be a reasonable alternate set of state.In novel differential equation appears less nonlinear, In adding together, the ballistic coefficients mean derived necessity exist zero. The unoriginal of the inverse ballistic coefficient be obliged toward be zero ${ }^{3,4}$. The genuine estimation of beta coefficient (i.e. mutual of inverse beta) actual ballistic coefficient, the 10 seconds, extremely accurate estimates can be obtained.Considering the altitude as $\mathrm{A}$, velocity as $\mathrm{V}$ and beta coefficient as $\mathrm{BC}$,Taylor-series as TS.

\section{Mathematical Formulation}

The object being tracked is unknown, so that it can be made a condition. In these situations, it determination indispensable in the direction of guesstimate the $\mathrm{A}, \mathrm{V}$, and $\mathrm{BC}$ of the object in classify in the direction of path. Also Considering

$$
S=\left[\begin{array}{l}
S \\
\dot{S} \\
1 / \beta
\end{array}\right]
$$

*Author for correspondence 
Where $s=$ state vector,the increase of rate performing on the object is a nonlinear role of the position according toward

$$
\ddot{S}=\left(\frac{1}{\beta}\right) \frac{0.0034 e^{-\mathrm{x} / 22000} \dot{s}^{2} g}{2}-g
$$

that the beta coefficient is unvarying, that means its derived must subsist zero ${ }^{5}$.

$$
\dot{1} / \beta=0
$$

Lineariza the preceding differential equations are given below

$$
\left[\begin{array}{c}
\Delta \dot{s} \\
\Delta \ddot{s} \\
\Delta(\dot{1} / \beta)
\end{array}\right]=\left[\begin{array}{ccc}
\frac{\partial \dot{s}}{\partial s} & \frac{\partial \dot{s}}{\partial \dot{s}} & \frac{\partial \dot{s}}{\partial(1 / \beta)} \\
\frac{\partial \ddot{s}}{\partial s} & \frac{\partial \ddot{s}}{\partial \dot{s}} & \frac{\partial \ddot{s}}{\partial \beta} \\
\frac{\partial(\dot{1} / \beta)}{\partial s} & \frac{\partial(\dot{1} / \beta)}{\partial \dot{s}} & \frac{\partial(1 / \beta)}{\partial(1 / \beta)}
\end{array}\right]\left[\begin{array}{c}
\Delta s \\
\Delta \dot{s} \\
\Delta(1 / \beta)
\end{array}\right]+\left[\begin{array}{c}
0 \\
0 \\
u_{s}
\end{array}\right]
$$

From the prior state-space degree of difference equation the system energetic pattern be the origin of partially derived

$$
F=\left[\begin{array}{ccc}
\frac{\partial \dot{s}}{\partial s} & \frac{\partial \dot{s}}{\partial \dot{s}} & \frac{\partial \dot{s}}{\partial(1 / \beta)} \\
\frac{\partial \ddot{s}}{\partial s} & \frac{\partial \ddot{s}}{\partial \dot{s}} & \frac{\partial \ddot{s}}{\partial(1 / \beta)} \\
\frac{\partial(\dot{1} / \beta)}{\partial s} & \frac{\partial(\dot{1} / \beta)}{\partial \dot{s}} & \frac{\partial(\dot{1} / \beta)}{\partial(1 / \beta)}
\end{array}\right]_{s=\hat{s}}
$$

Here it can observe with the purpose of partially derived as a result of calculate approximately from the current state guesstimate. Observed the linearity statespace equation from the incessant development-noise pattern is given by.

$$
\mathrm{Q}=\mathrm{E}\left[\mathrm{ww} \mathrm{w}^{\mathrm{T}}\right]
$$

Therefore, the incessant process-noise pattern is

$$
Q(t)=\left[\begin{array}{ccc}
0 & 0 & 0 \\
0 & 0 & 0 \\
0 & 0 & \Phi_{s}
\end{array}\right]
$$

Where $\phi_{s}$ is a white noise spectral density is assumed in the direction of the beta coefficient ${ }^{6,7}$ is evaluate from the dynamics system pattern,

$$
\begin{aligned}
& \frac{\partial \dot{s}}{\partial s}=0 \\
& \frac{\partial \dot{s}}{\partial \dot{s}}=1 \\
& \frac{\partial \dot{s}}{\partial(1 / \beta)}=0
\end{aligned}
$$

the essential partial derivatives yields from the nonlinear situation equation is observed from both $1^{\text {st }}$ row and $2^{\text {nd }}$ row .

$$
\begin{aligned}
& \frac{\partial \ddot{s}}{\partial \mathrm{s}}=\frac{-0.0034 \mathrm{e}^{-x / 22000} \dot{\mathrm{s}}^{2} \mathrm{~g}}{2 \beta(22,000)}=\left(\frac{1}{\beta}\right) \frac{-\rho \dot{\mathrm{s}}^{2} \mathrm{~g}}{44,000 \beta} \\
& \frac{\partial \ddot{\mathrm{s}}}{\partial \dot{\mathrm{s}}}=\frac{2 * 0.0034 \mathrm{e}^{-x / 22000} \dot{\mathrm{s} g}}{2 \beta}=\left(\frac{1}{\beta}\right) \frac{\rho \dot{\mathrm{s} g}}{\beta} \\
& \frac{\partial \ddot{\mathrm{s}}}{\partial 1 / \beta}=\frac{-0.0034 \mathrm{e}^{-x / 22000} \dot{\mathrm{s}}^{2} \mathrm{~g}}{2 \beta^{2}}=\frac{-\rho \dot{\mathrm{s}}^{2} \mathrm{~g}}{2 \beta^{2}}
\end{aligned}
$$

the preceding row of partial derivatives acquiesces

$$
\begin{gathered}
\frac{\partial(1 \dot{\beta})}{\partial s}=0 \\
\frac{\partial(1 / \beta)}{\partial \dot{s}}=0 \\
\frac{\partial(1 / \beta)}{\partial(1 / \beta)}=0 \\
F=\left[\begin{array}{ccc}
0 & 1 & 0 \\
\frac{-p \dot{s}^{2} g}{44}, 000(1 / \beta) & \frac{\rho \dot{s} g}{1 / \beta} & \frac{-p \dot{s}^{2} g}{2\left(\frac{1}{\beta}\right)^{2}} \\
0 & 0 & 0
\end{array}\right]
\end{gathered}
$$

where $g$ is tacit to subsist identified gravity entirely, excluding the inverse beta coefficient $\left(\frac{1}{\beta}\right)$ ought to be guesstimate. The expected atmosphere compactness during the earlier appearance depends on the projected $\mathrm{A}^{8}$.

$$
\hat{\rho}=0.0034 e^{-\mathrm{x} / 22000}
$$

The essential pattern know how to be originate through TS approximately 9 . 


$$
\phi(t)=\mathrm{e}^{\mathrm{Ft}}=\mathrm{I}+\mathrm{Ft}+\frac{(\mathrm{Ft})^{2}}{2 !}+\cdots+\frac{(\mathrm{Ft})^{\mathrm{n}}}{\mathrm{n} !}+\cdots
$$

By defining as

$$
\begin{aligned}
& f_{21}=\frac{-p \dot{s}^{2} g}{44}, 000(1 / \beta) \\
& f_{22}=\frac{\rho \dot{s} g}{1 / \beta} \\
& f_{23}=\frac{-p \dot{s}^{2} g}{2\left(\frac{1}{\beta}\right)^{2}}
\end{aligned}
$$

the dynamics pattern and original rough calculation given by

$$
\begin{gathered}
\mathrm{F}(\mathrm{t})=\left[\begin{array}{ccc}
0 & 1 & 0 \\
f_{21} & f_{22} & f_{23} \\
0 & 0 & 0
\end{array}\right] \\
\varphi_{\mathrm{k}}=\left[\begin{array}{ccc}
0 & 1 & 0 \\
f_{21} T_{s} & 1+f_{22} T_{s} & f_{23} T_{s} \\
0 & 0 & 0
\end{array}\right]
\end{gathered}
$$

\section{Analysis by Compression}

From figures 1 and 3 by observeing the simulation results take place in the guesstimates of $\mathrm{A}, \mathrm{V}$ and $\mathrm{BC}$ come into view the process noise is zero. It is observed that the error in the estimation of altitude is within the bound. Figures 2 and 3 indicates the hanging off portion errors while estimating of velocity and beta coefficient. That there is a dissimilarity among the covariance matrices, is being observing the actually position is achieved. There is rejection for process noise errors within the estimation. There must be continuous reduction at the same time more measurements are taken through small errors in the estimation, the errors in estimation of ballistic coefficient 10 seconds earlier than figure 2 have been achieved.

From figures 4 and 3 it is observed that an error in the guesstimate of $\mathrm{BC}$ reduces since moment in time expanded (A reduces) and more hang off errors occurring. From the figures 5 and 1 it is seen that there is no process noise and there is no extensive for any hang off error in the inaccuracy in the guesstimate of $\mathrm{V}$ and also there is refusal noise occurred. From figure 6 representing, there is no hang off error in the error of estimating

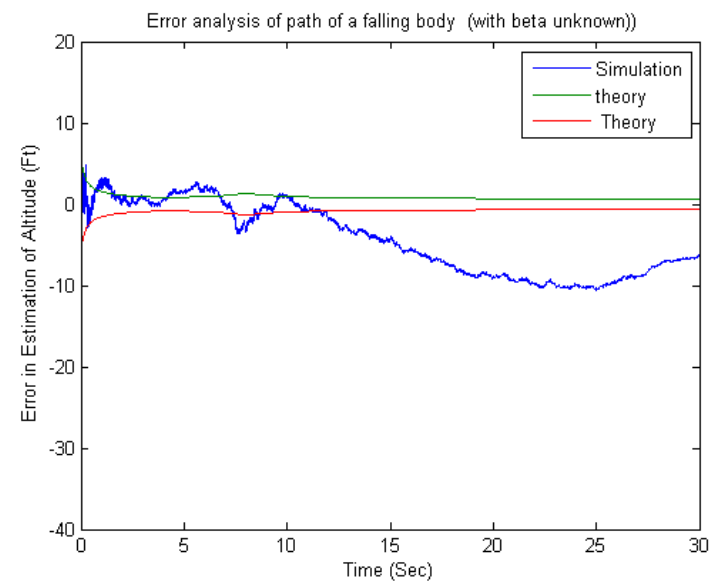

Figure 1. Error estimation in altitude.

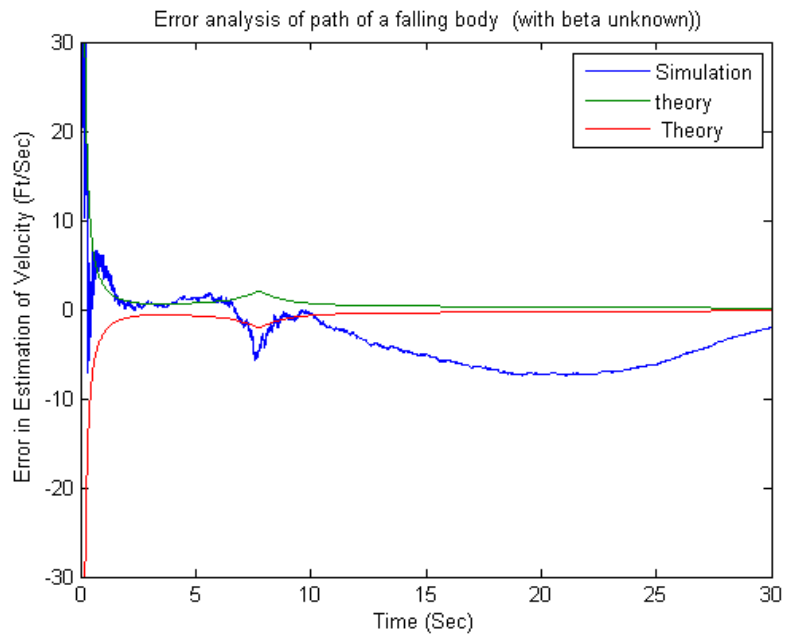

Figure 2. Error estimation in velocity.

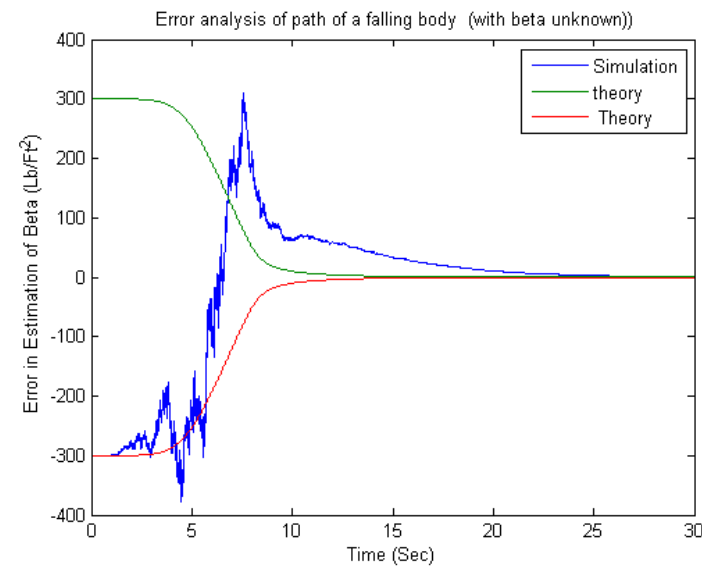

Figure 3. Error analysis for falling body with unkown beta. inverse beta coefficients. Also it can be observed with the aim of the inverse beta coefficient is not apparent 


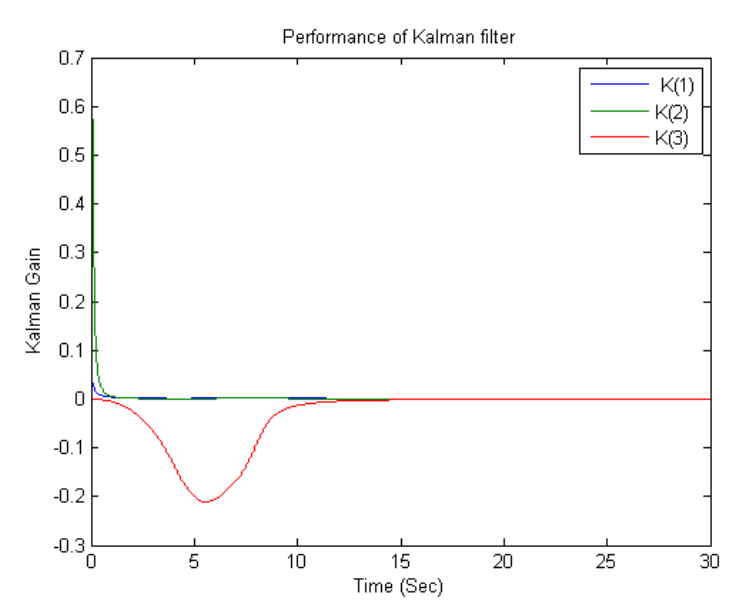

Figure 4. Performance of Kalman filter in estimating hang off error in beta coefficient.

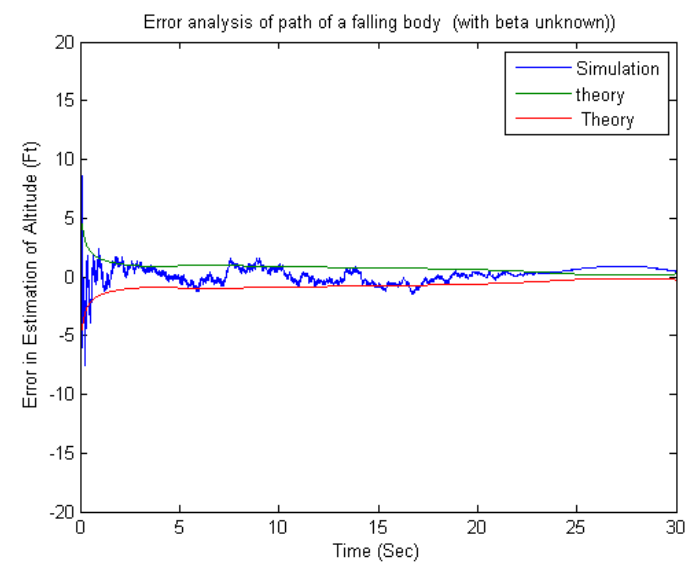

Figure 5. Altitude error analysis of path for unknown beta falling object.

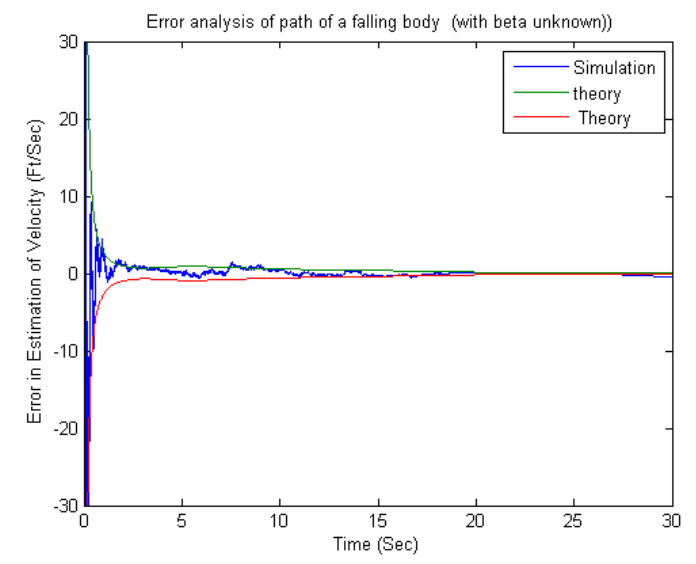

Figure 6. Velocity error analysis of path for unknown beta falling object. at the equivalent stage of time as in (EKF) from figure 3. Comparing the results from the figure 7 more clarity is observed now that hang off errors have occurred. Now, finally comparing the results of figure 4 and figure 8 (showing that figure 8 has more accurate results than figure 4), accurate estimates within the period of $10 \mathrm{sec}$ onds are obtained.

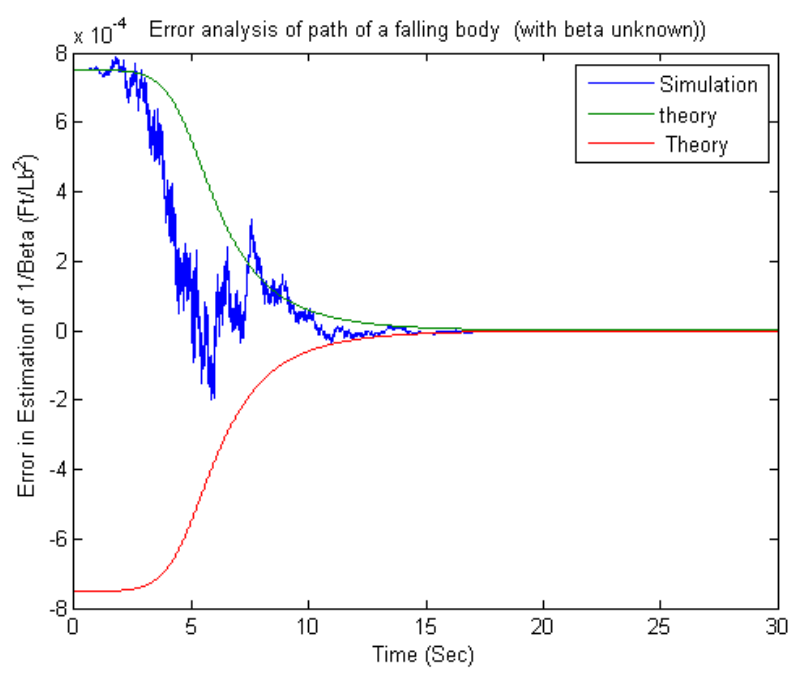

Figure 7. Inverse beta error analysis of path for unknown beta falling object.

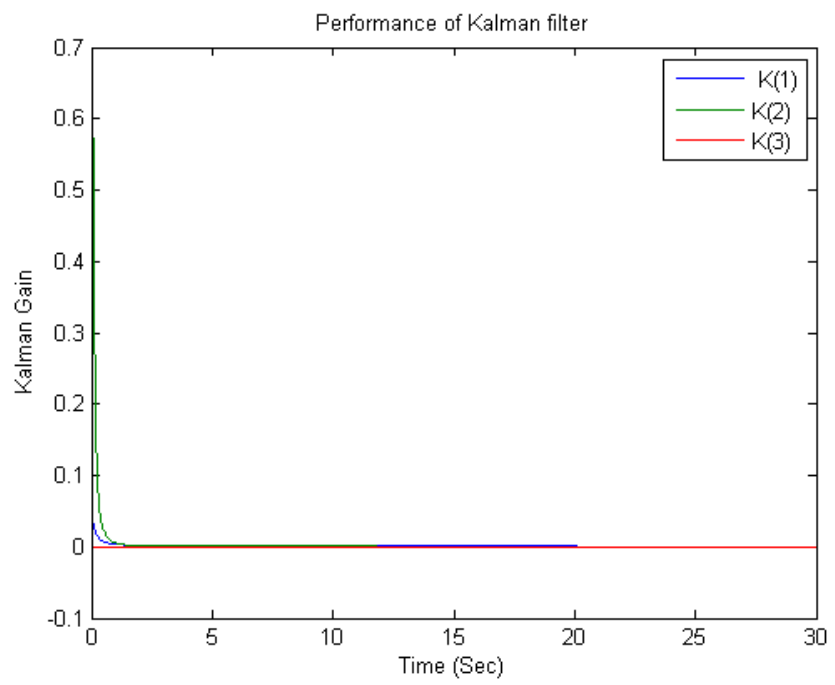

Figure 8. Accurate Kalman gain observed through unknown beta for falling object.

\section{Interpretation}

All the comparsions prove beyond doubt the effectiveness of $\left(\frac{1}{\beta}\right)$ factor. More over limitation of period to 10 
seconds has a lot of pratical importance.These observations of compression can construct the filter more amnable in the direction of unexpected changes in the object ${ }^{10}$. Elimination of process noise and hang off error can make an idealized optimization of the filter.

\section{Acknowledgment}

Authors would like to thank Principal and Management of Vignana's Institute of Information Technology for extended their support in completion of the work.

\section{Conclusion}

In this paper an analysis of effect of ballistic coefficient and performance of EKF with emphasis on air drag is discussed and the falling object problem for the effectiveness of adding up development noise to EKF to eliminate hang off error in the approximations to formulate the filter supplementary to unexpected changes in the object's dynamics. Change the state of an EKF can sometime reduces estimation errors for the reason that firm state makes the position appear a smaller amount non linear. The goal has been to path for the object and guesstimate $\mathrm{A}, \mathrm{V}$, and speeding up also calculate approximately. The object's BC in proving the effectiveness of our assumption, this work has been secessfully used to radar tracking applications.

\section{References}

1. Zarchan P, Musoff H. Fundamentals of Kalman Filtering: A Practical Approach. 3rd ed. American Institute of Aeronautics and Astronautics; 2009.
2. Arulampalam M. A tutorial on particle filters for online nonlinear/non-Gaussian Bayesian tracking. IEEE Transaction on Signal Processing. 2002; 50(2):174-88. ISSN: 1053-587X.

3. Dey A. Adaptive divided difference filter for parameter and state estimation of non-linear systems. IEEE International Conference on Control, Instrumentation, Energy and Communication. 2014; 9(4):369-76.

4. Stone LD. Multiple target tracking with quantized measurements: A standard Bayesian approach. IEEE 17th International Conference on Information Fusion (FUSION); 2014 July. p. 1-8.

5. Pedersen RN. System and method for detecting use of booster rockets by ballistic missiles. 2015 .

6. Shin SJ. Re-entry vehicle tracking with a new multiple model estimation applicable to highly non-linear dynamics. 2015 June; 9(5):581-8.

7. Hong-De D, Shao-Wu D, Yuan-Cai C, Guang-Bin W. Performance Comparison of EKF/UKF/CKF for the Tracking of Ballistic Target. TELKOMNIKA Indonesian Journal of Electrical Engineering. 2012; 10(7):1692-9.

8. George S, Vasile V, Slamnoiu G. Applications of probability theory to study the influence of projectile's mass tolerances on exterior ballistic. Review of the Air Force Academy. 2014; 3(27):101-4.

9. Altaf A, Raeisi A. Presenting an effective algorithm for tracking of moving object based on support vector machine. Indian Journal of Science and Technology. 2015; 8(17):1-7.

10. Safarinejadian B, Mozaffari M. A new Kalman filter based state estimation method for multi-input multi-output unit time-delay systems. Indian Journal of Science and Technology. 2013; 6(3):4205-12. 